\title{
FUNCTION THEORETIC METHODS FOR HIGHER ORDER, ELLIPTIC EQUATIONS IN THREE INDEPENDENT VARIABLES ${ }^{1}$
}

\author{
BY R. P. GILBERT AND D. KUKRAL \\ Communicated by H. F. Weinberger, May 18, 1972
}

Introduction. S. Bergman [1] and I. N. Vekua [5] have developed function theoretic methods for treating analytic, elliptic equations in two variables. In particular, they have developed integral operator methods for the normalized, second order equation

$$
\Delta u+a u_{x}+b u_{y}+c u=0
$$

and the fourth order equation

$$
\Delta \Delta u+a u_{x x}+2 b u_{x y}+c u_{y y}+d u_{x}+e u_{y}+f u=0 .
$$

Colton [2], [3] has shown recently how one may extend the results of Bergman and Vekua for the second order case when the coefficients and solution are to be dependent on three variables. Colton's method is based on some earlier work of Tjong [4].

In this note we wish to announce that one may extend the ideas used by Colton and Tjong to treat also equations of higher order, which depend on three independent variables. We remark that this is the first time a function theoretic method has been developed for a fairly general, higher order equation in three independent variables. To simplify our presentation, and because of lack of space we will announce our results merely for the case

$$
\Delta \Delta u+Q(x, y, z) u=0
$$

the more general case, corresponding to equations (2) and the higher order analogues may be treated in the same manner.

The generating kernels. The approach used by Bergman and Vekua has been to continue the elliptic equation into the complex domain where it is formally hyperbolic. By introducing the variables $X=x, Z=\frac{1}{2}[y+i z]$,

AMS (MOS) subject classifications (1970). Primary 35A20, 35C15; Secondary 35J15.

Key words and phrases. Integral operators, elliptic equation, analytic functions, complete families.

${ }^{1}$ Research sponsored by the Air Force Office at Scientific Research Air Force Systems Command, USAF, under AFOSR grants 1206-67 and 71-2205A. The United States Government is authorized to reproduce and distribute reprints for Government purposes notwithstanding any copyright notation hereon. 
$Z^{*}=\frac{1}{2}[-y+i z]$ equation (3) may be written in the form

$$
\frac{\partial^{4} U}{\partial Z^{2} \partial Z^{* 2}}-2 \frac{\partial^{4} U}{\partial X^{2} \partial Z \partial Z^{*}}+\frac{\partial^{4} U}{\partial X^{4}}+Q\left(X, Z, Z^{*}\right) U=0 .
$$

It is convenient for our exposition to introduce the variables [4], [2]

$$
\xi_{1}=2 \zeta Z, \quad \xi_{2}=X+2 \zeta Z, \quad \xi_{3}=X+2 \zeta^{-1} Z^{*},
$$

where $\zeta$ is a complex variable such that $1-\varepsilon<|\zeta|<1+\varepsilon, 0<\varepsilon<\frac{1}{2}$. Furthermore, let $E^{*}\left(\xi_{1}, \xi_{2}, \xi_{3}, \zeta, t\right) \equiv E\left(X, Z, Z^{*}, \zeta, t\right)$, and $E_{i}^{*} \equiv \partial E^{*} / \partial \xi_{i}$ $(i=1,2,3), E_{t}^{*} \equiv \partial E^{*} / \partial t$. Suppose $E^{*}$ is a solution of the partial differential equation

$$
\begin{aligned}
E_{2222}^{*} & +E_{3333}^{*}+6 E_{2233}^{*}-8 E_{1223}^{*}-4 E_{2223}^{*} \\
& -8 E_{1333}^{*}-4 E_{2333}^{*}+16 E_{1133}^{*}+16 E_{1233}^{*} \\
& -\frac{2\left(1-t^{2}\right)}{\mu t}\left(E_{122 t}^{*}+E_{133 t}^{*}-4 E_{113 t}^{*}-2 E_{123 t}^{*}\right) \\
& +\frac{2}{\mu t^{2}}\left(E_{122}^{*}+E_{133}^{*}-4 E_{113}^{*}-2 E_{123}^{*}\right) \\
& +\frac{1}{\mu^{2} t^{2}}\left(1-t^{2}\right)^{2} E_{11 t t}^{*}+\frac{E_{11 t}^{*}}{t}\left(-3+3 t^{4}\right)+\frac{3 E_{11}^{*}}{t^{2}} \\
& +Q^{*} E^{*}=0,
\end{aligned}
$$

for $(x, y, z) \in \mathfrak{G} \subset C^{3}$, where $\mathfrak{b}$ is some neighborhood of the origin, $|t| \leqq 1$, and $\zeta$ is in the annulus described above. Then

$$
U\left(X, Z, Z^{*}\right)=\frac{1}{2 \pi i} \int_{|\zeta|=1} \int_{\gamma} E\left(X, Z, Z^{*}, \zeta, t\right) f(\omega, \zeta) \frac{d t}{\left(1-t^{2}\right)^{1 / 2}} \frac{d \zeta}{\zeta},
$$

where $\gamma$ is a path joining $t=-1$ and $t=+1$, is a (complex valued) solution of equation (4) which is regular in a neighborhood of the origin in $X, Z, Z^{*}$ space.

One may prove that solutions of (5) exist using the method of majorants. Indeed, we may find two solutions of the form

$$
\begin{aligned}
& E^{*}\left(\xi_{1}, \xi_{2}, \xi_{3}, \zeta, t\right)=1+\sum_{n=1}^{\infty} t^{2 n} \mu^{n} p^{(n)}\left(\xi_{1}, \xi_{2}, \xi_{3}, \zeta\right), \\
& \hat{E}^{*}\left(\xi_{1}, \xi_{2}, \xi_{3}, \zeta, t\right)=\frac{\xi_{1}}{2 \zeta}+\sum_{n=1}^{\infty} t^{2 n} \mu^{n} q^{(n)}\left(\xi_{1}, \xi_{2}, \xi_{3}, \zeta\right),
\end{aligned}
$$

where the coefficients $p^{(n)}\left(\xi_{1}, \xi_{2}, \xi_{3}, \zeta\right)$ and $q^{(n)}\left(\xi_{1}, \xi_{2}, \xi_{3}, \zeta\right)$ are uniquely determined by the following: 


$$
\begin{aligned}
& p^{(1)}\left(\xi_{1}, \xi_{2}, \xi_{3}, \zeta\right)=0=q^{(1)}\left(\xi_{1}, \xi_{2}, \xi_{3}, \zeta\right), \\
& p_{11}^{(2)}\left(\xi_{1}, \xi_{2}, \xi_{3}, \zeta\right)=-\frac{1}{3} Q^{*}\left(\xi_{1}, \xi_{2}, \xi_{3}, \zeta\right), \\
& q_{11}^{(2)}\left(\xi_{1}, \xi_{2}, \xi_{3}, \zeta\right)=-\frac{1}{3}\left(\xi_{1} / 2 \zeta\right) Q^{*}\left(\xi_{1}, \xi_{2}, \xi_{3}, \zeta\right),
\end{aligned}
$$

and for $n \geqq 1$ both $p^{(n)}$ and $q^{(n)}$ satisfy

$$
\begin{aligned}
p_{11}^{(n+2)}=\frac{1}{(2 n+1)(2 n+3)}\{ & 2(2 n+1)\left(p_{122}^{(n+1)}+p_{133}^{(n+1)}-4 p_{133}^{(n+1)}-2 p_{123}^{(n+1)}\right) \\
& -p_{2222}^{(n)}-p_{3333}^{(n)}-6 p_{2233}^{(n)}+4 p_{2223}^{(n)}+4 p_{2333}^{(n)} \\
& +8 p_{1223}^{(n)}+8 p_{1333}^{(n)}-16 p_{1233}-16 p_{1133} \\
& \left.-Q^{*} p^{(n)}\right\},
\end{aligned}
$$

such that, for $n=1,2, \ldots$,

$$
\begin{aligned}
& p^{(n)}\left(0, \xi_{2}, \xi_{3}, \zeta\right)=0=q^{(n)}\left(0, \xi_{2}, \xi_{3}, \zeta\right), \\
& p_{1}^{(n)}\left(0, \xi_{2}, \xi_{3}, \zeta\right)=0=q_{1}^{(n)}\left(0, \xi_{2}, \xi_{3}, \zeta\right) .
\end{aligned}
$$

Complete families of solutions. The particular choice of the generating kernels indicated by (7) are of importance in showing that representations of the form

$$
\begin{aligned}
& u(x, y, z)= \operatorname{Re} \boldsymbol{P}_{3}^{(2)}\{f, \hat{f}\}, \\
& \boldsymbol{P}_{3}^{(2)}\{f, \hat{f}\} \equiv \frac{1}{2 \pi i} \int_{|\zeta|=1} \int_{\gamma}\left[E\left(X, Z, Z^{*}, \zeta, t\right) f(\omega, \zeta) \frac{d t}{\left(1-t^{2}\right)^{1 / 2}} \frac{d \zeta}{\zeta}\right. \\
&\left.+\hat{E}\left(X, Z, Z^{*}, \zeta, t\right) \hat{f}(\omega, \zeta) \frac{d t}{\left(1-t^{2}\right)^{1 / 2}} \frac{d \zeta}{\zeta}\right],
\end{aligned}
$$

are maps of pairs of holomorphic functions onto $\mathscr{C}^{4}$, real solutions of (3). Indeed, we are able to prove the

THEOREM. Let $u(x, y, z)$ be a real valued $\mathscr{C}^{4}$ solution of equation (3) in some neighborhood of the origin in $\boldsymbol{R}^{3}$. Then there exists a pair of analytic functions of two complex variables $\{f(\mu, \zeta), \hat{f}(\mu, \zeta)\}$ which are regular for $\mu$ in some neighborhood of the origin and $|\zeta|<1+\varepsilon, \varepsilon>0$, such that locally $u(x, y, z)=\operatorname{Re} \boldsymbol{P}_{3}^{(2)}\{f, \hat{f}\}$.

In particular, denote by $U\left(X, Z, Z^{*}\right)$ the extension of $u(x, y, z)$ to the $X, Z, Z *$ space and let

$$
\begin{aligned}
& f(\mu, \zeta)=-\frac{1}{2 \pi} \int_{\gamma^{\prime}} g\left(\mu\left(1-t^{2}\right), \zeta\right) d t / t^{2}, \\
& \hat{f}(\mu, \zeta)=-\frac{1}{2 \pi} \int_{\gamma^{\prime}} \hat{g}\left(\mu\left(1-t^{2}\right), \zeta\right) d t / t^{2},
\end{aligned}
$$


where $\gamma^{\prime}$ is a rectifiablc arc joining the points $t=-1$ and $t=+1$ and not passing through the origin. Here

$g(\mu, \zeta)=2 \frac{\partial}{\partial \mu}\left[\mu \int_{0}^{1} U(t \mu, 0,(1-t) \mu \zeta) d t\right]-U(\mu, 0,0)$,

$\hat{g}(\mu, \zeta)=2 \frac{\partial}{\partial \mu}\left[\mu \int_{0}^{1} U_{2}(t \mu, 0,(1-t) \mu \zeta) d t\right]$

$$
\begin{aligned}
& -\zeta \frac{\partial}{\partial \mu}\left[g(\mu, \zeta)-\frac{1}{2 \pi i} \int_{|\alpha|=1} g(\mu / \alpha, \zeta \alpha) \frac{d \alpha}{\alpha}-\frac{1}{2 \pi i} \int_{|\alpha|=1} g(u / 2, \zeta \alpha) d \alpha\right] \\
& +g_{\zeta \mu}(\mu, 0)-\zeta \int_{0}^{\mu} U_{\zeta}(\mu, 0,0) d \mu .
\end{aligned}
$$

The previous theorem implies that we may generate a family of solutions with the Runge approximation property.

THEOREM III. Let $\mathbf{G}$ be a bounded simply connected domain in $\boldsymbol{R}^{3}$, and define

$$
\begin{aligned}
U_{4 n, m} & =\operatorname{Re} P_{3}^{(2)}\left\{\mu^{n \zeta^{m}}, 0\right\}, & & 0 \leqq n<\infty, m=0, \ldots, n, \\
U_{4 n+1, m} & =\operatorname{Re} P_{3}^{(2)}\left\{0, \mu^{n} \zeta^{m}\right\}, & & 0 \leqq n<\infty, m=0, \ldots, n, \\
U_{4 n+2, m} & =\operatorname{Im} P_{3}^{(2)}\left\{\mu^{n} \zeta^{m}, 0\right\}, & & 0 \leqq n<\infty, m=0, \ldots, n, \\
U_{4 n+3, m} & =\operatorname{Im} P_{3}^{(2)}\left\{0, \mu^{n} \zeta^{m}\right\}, & & 0 \leqq n<\infty, m=0, \ldots, n,
\end{aligned}
$$

where "Im" denotes "take the imaginary part". Then the set $\left\{U_{n, m}\right\}$ is a complete family of solutions for equation (3) in the space of real valued $\mathscr{C}^{4}$ solutions of $(3)$ defined in $(\mathfrak{G}$.

\section{REFERENCES}

1. S. Bergman, Integral operators in the theory of linear partial differential equations, Ergebnisse der Mathematik und ihre Grenzgebiete, N. F., Heft 23, Springer-Verlag, Berlin, 1961. MR 25 \# 5277.

2. D. Colton, Bergman operators for elliptic equations in three independent variables, Bull. Amer. Math. Soc. 77 (1971), 752-756. MR 43 \#6578.

3. - Integral operators for elliptic equations in three independent variables. I, II, Applicable Anal. (to appear).

4. B. L. Tjong, Operators generating solutions of $\Delta_{3} \psi(x, y, z)+F(x, y, z) \psi(x, y, z)=0$ and their properties, Analytic Methods in Math. Physics, edited by R. P. Gilbert and R. G. Newton, Gordon and Breach, New York, 1970.

5. I. N. Vekua, New methods for solving elliptic equations, OGIZ, Moscow, 1948; English transl., Series in Appl. Math., vol. 1, North-Holland, Amsterdam, Interscience, New York, 1967, MR 11, 598; MR 35 \#3243.

Department of Mathematics, Indiana University, Bloomington, Indiana 47401

Current address (R. P. Gilbert): Department of Mathematics, University of Delaware, Newark, Delaware 19711

Current address (D. Kukral): Department of Mathematics, Wichita State University, Wichita, Kansas 67208 